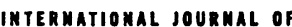

\section{Size-dependent rigidities of nanosized torsional elements}

\author{
Vijay B. Shenoy * \\ Materials Research Centre, Indian Institute of Science, Bangalore, 560 012, India
}

Received 23 June 2001; received in revised form 5 March 2002

\begin{abstract}
A theory for the prediction of the size dependence of torsional rigidities of nanosized structural elements is developed. It is shown that, to a very good approximation, the torsional rigidity $(D)$ of a nanosized bar differs from the prediction of standard continuum mechanics $\left(D_{\mathrm{c}}\right)$ as $\left(D-D_{\mathrm{c}}\right) / D_{\mathrm{c}}=A h_{0} / a$ where $A$ is a non-dimensional constant, $a$ is the size scale of the cross-section of the bar and $h_{0}$ is a material length equal to the ratio of the surface elastic constant to the bulk elastic constant. The theory developed is compared with direct atomistic calculations ("numerical experiment") of the torsional rigidity bars made of several FCC metals modeled using the embedded atom method. Very good agreement is obtained between theory and simulation. The framework presented here can aid the development of design methodologies for nanoscale structural elements without the need for full scale atomistic simulations. () 2002 Published by Elsevier Science Ltd.
\end{abstract}

Keywords: Nanostructures; Elastic properties; Atomistic modeling; Surface energy

\section{Introduction}

The demand for smaller and faster devices have encouraged technological advances resulting in the ability to manipulate matter at micro and nanoscales that have enabled the fabrication of micro/nanoscale electromechanical systems. While MEMS technology is now a well established area, nanoelectromechanical systems (NEMS) have recently made their appearance in literature (see, for example, Roukes, 2000). A key feature of NEMS that make them attractive are their high fundamental frequencies while affording small force constants. Nanosized bars and tubes are produced in a variety of ways with materials such as $\mathrm{SiC}$, $\mathrm{MoO}_{3}$ and $\mathrm{C}$ (carbon nanotubes) (see Yakobson and Smalley, 1997; Terrones et al., 1999; Sheehan and Lieber, 1996). These nanosized elements have found many technological uses, for example, as probes of scanning probe microscopes (Dai et al., 1996), in altering properties of bulk materials in the form of whisker additions (Kuzumake et al., 1998) etc.

In the recent past, several groups have reported studies on the mechanical behaviour nanosized bars and nanotubes. Wong et al. (1997) performed experiments on SiC beams while Poncharal et al. (1999), and more recently Gao et al. (2000) have reported experiments where the elastic modulus of carbon nanotubes is

\footnotetext{
${ }^{*}$ Fax: +91-80-360-0683.

E-mail address: shenoy@mrc.iisc.ernet.in (V.B. Shenoy).
} 
measured using dynamic techniques. A careful study of these reports show that the elastic moduli of such nanosized structural elements depend on their size. Attempts to explain the size dependent behaviour has been through direct atomistic computer simulation of these structures (Robertson et al., 1992; Garg and Sinnott, 1998; Garg et al., 1998). These studies have reported size-dependent elastic moduli computed from atomistic simulations. Similar studies of shape dependence of properties have also been reported on nanoparticles (Yannouleas et al., 2001).

Miller and Shenoy (2000) developed a simple model to explain the size dependence of the elastic rigidities of nanosized structural elements. These size dependences were attributed to the heterogeneities in the atomic environments introduced by the bounding free surfaces of the structural elements. Thus, as the size of the structure becomes smaller the presence of surfaces have to be accounted for in modeling strategies. Developing this premise, Miller and Shenoy (2000) showed that the differences between the rigidities $(D)$ of these small elements and those predicted by continuum mechanics $\left(D_{\mathrm{c}}\right)$ can be expressed as

$$
\frac{D-D_{\mathrm{c}}}{D_{\mathrm{c}}}=A \frac{h_{0}}{a}
$$

where $A$ is a non-dimensional constant that depends on the geometry of the structure, $a$ is the size scale of the structural element (for example, the cross-sectional width of a bar), and $h_{0}$ is a material length that is the ratio of the surface elastic constant of the bounding surfaces of the structure and the bulk elastic constant of the material. Thus, the size dependence of the rigidities can be predicted by obtaining the material parameter $h_{0}$ and the non-dimensional constant $A$. Typically, $h_{0}$ can be obtained from a small atomistic simulation and $A$ can be calculated analytically. Thus the need for full scale atomistic simulations of structures (which is an expensive proposition) is obviated. Miller and Shenoy (2000) applied this model to study the elastic properties of nanosized bars, plates and beams and demonstrated the strength of the model by comparison with direct atomistic calculations.

An important mode of deformation of bar-like structures is torsion; for example, the probe of a scanning probe microscope is subjected to bending and torsion. It is therefore important to develop a model for the torsional rigidities of nanosized elements; this is the aim of the present paper. An augmented continuum theory of torsion accounting for the presence of free surfaces is developed and the size dependence of the rigidity is derived analytically. A perturbative scheme is developed for solving the resulting boundary value problem which provides a simple method to evaluate the size dependence of the torsional rigidity. It is shown that, in general, the size dependence of the rigidity $D$ is of the form

$$
\frac{D-D_{\mathrm{c}}}{D_{\mathrm{c}}}=A \frac{h_{0}}{a}+B\left(\frac{h_{0}}{a}\right)^{2}+\cdots
$$

where $A, B$, etc. are non-dimensional constants and $h_{0}=S / G$ where $S$ is the surface shear modulus and $G$ is the bulk shear modulus. The perturbative method also provides a general framework for the calculation of the non-dimensional constants $A, B, \ldots$ that depend only on the geometry of the cross-section of the bar. For the case of square bars of side $2 a$, the theory provides that size dependence of torsional rigidity to be very well approximated by

$$
\frac{D-D_{\mathrm{c}}}{D_{\mathrm{c}}} \approx 4 \frac{h_{0}}{a}
$$

These theoretical results are then compared with direct atomistic simulations (which serve as numerical experiments) of torsion of square bars of FCC metals. The methodology required for the atomistic simulation of torsion is also developed here. The agreement theory and numerical experiment (atomistic simulations) is excellent. 
The paper is organised as follows. Section 2 contains the augmented theory of torsion. Section 3 contains the details of simulation methodologies, the results of which are reported and discussed in Section 4 . The paper in concluded in Section 5 in which several important directions of future work are identified.

\section{Theory}

\subsection{Augmented continuum theory}

It was shown by Miller and Shenoy (2000) that the elastic properties of nanoscale structural elements such as plates, bars and beams can be explained using a augmented continuum theory that accounts for the energetics of deforming inhomogeneities such as surfaces and corners, the effects of which are significant when the length scale of the structure approaches the atomic scale. Several authors had previously utilized continuum theories of solids with surface effects (Gurtin and Murdoch, 1975; Rice and Chuang, 1981; Cammarata, 1994) to study a variety of problems ranging from diffusive cavity growth in stressed solids to stability of stressed epitaxial films. The formulation outlined in Miller and Shenoy (2000) is briefly recapitulated here for the sake of completeness and to set the notation.

The body $\mathscr{B}$, described by coordinates $x_{i}$, considered in the augmented continuum theory is bounded by a surface $\mathscr{S}$. It is assumed that the surface $\mathscr{S}$ is piecewise flat (this assumption eliminates the need to consider contravariant and covariant components of surface tensors) and is described by coordinates $x_{\alpha}$ for each flat face. The bulk stress tensor in the body $\mathscr{B}$ is denoted by $\sigma_{i j}$ and the surface stress tensor by $\tau_{\alpha \beta}$. Mechanical equilibrium of a bulk material element implies that the bulk stress tensor satisfies (with no body forces)

$$
\sigma_{i j, j}=0
$$

Equilibrium of a surface element necessitates that

$$
\begin{gathered}
\tau_{\alpha \beta, \beta}+f_{\alpha}=0 \\
\tau_{\alpha \beta} \kappa_{\alpha \beta}=\sigma_{i j} n_{i} n_{j}
\end{gathered}
$$

where $n_{i}$ is the outward normal to the surface, $f_{\alpha}$ is the negative of the tangential component of the traction $t_{i}=\sigma_{i j} n_{j}$ along the $\alpha$ direction of surface $\mathscr{S}$, and $\kappa_{\alpha \beta}$ is the surface curvature tensor. The assumption of the piecewise flat surfaces implies that the surface curvature vanishes everywhere along the surface except at corners and edges which have to be treated separately. It must be noted that the assumption of piecewise flat surface is merely for the sake of mathematical simplicity; the present theoretical framework is valid for curved surfaces as well.

The kinematics of the body is described by the displacement field $u_{i}$ defined at every point in the body. The strain tensor $\epsilon_{i j}$ in the body is obtained using a small strain formulation as

$$
\epsilon_{i j}=\frac{1}{2}\left(u_{i, j}+u_{j, i}\right)
$$

The surface strain tensor $\epsilon_{\alpha \beta}$ is derived from the bulk strain tensor $\epsilon_{i j}$ such that every material fibre on the surface has the same deformation whether it is treated as a part of the surface or as a part of the bulk, i.e., the surface strain tensor is compatible with the bulk strain tensor.

The final ingredient of the augmented continuum theory is the constitutive relations that relate the stresses to strains. The bulk is considered to be an anisotropic linear hyperelastic solid with a free energy density $W$ defined as

$$
W\left(\epsilon_{i j}\right)=\frac{1}{2} C_{i j k l} \epsilon_{i j} \epsilon_{k l}
$$


and the stresses are derived as

$$
\sigma_{i j}=\frac{\partial W}{\partial \epsilon_{i j}}=C_{i j k l} \epsilon_{k l}
$$

where $C_{i j k l}$ is the bulk elastic modulus tensor. In this framework the bulk free energy vanishes for the unstrained solid. Constitutive relations for the surface stress tensor are more involved. The surface stress tensor is related to the surface energy $\gamma$ as

$$
\tau_{\alpha \beta}=\gamma \delta_{\alpha \beta}+\frac{\partial \gamma}{\partial \epsilon_{\alpha \beta}},
$$

a relation which is generally attributed to Gibbs (Cammarata, 1994). The surface stress tensor can be expressed as a linear function of the strain tensor as

$$
\tau_{\alpha \beta}=\tau_{\alpha \beta}^{0}+S_{\alpha \beta \gamma \delta} \epsilon_{\gamma \delta}
$$

where $\tau_{\alpha \beta}^{0}$ is the surface stress tensor when the bulk is unstrained (obtained from (10) with $\epsilon_{\alpha \beta}=0$ ) and $S_{\alpha \beta \gamma \delta}$ is the surface elastic modulus tensor. This is an important quantity in that the size dependence of elastic properties will be shown to be determined by the ratio of a surface elastic constant and the bulk elastic constant. The constitutive constants $C_{i j k l}$ and $S_{\alpha \beta \gamma \delta}$ are external to the augmented continuum theory; in this paper these are determined from atomistic models of the materials considered.

\subsection{Augmented theory of torsion of bars}

The continuum theory of torsion of bars is a part of classical theory of elasticity attributed to St. Venant and Prandtl and is treated in much detail by Sokolnikoff (1956). In this section we develop a theory of torsion based on the augmented theory of last section which includes surface effects. Corner effects are neglected in this treatment.

Fig. 1 shows the cross-section of the bar $\mathscr{B}$ bounded by a surface $\mathscr{S}$. Attention is restricted to simply connected cross-sections for the sake of simplicity. The outward normal to $\mathscr{S}$ is denoted by $\boldsymbol{n}$ and $\boldsymbol{s}$ is the tangent vector to $\mathscr{S}$ with $\boldsymbol{n} \cdot \boldsymbol{s}=0$. Application of a torque $T$ to the bar produces a twist per unit length $\alpha$; the aim of the analysis is to obtain a relationship between $T$ and $\alpha$. The kinematics is described by the displacement field as

$$
\begin{aligned}
& u_{1}\left(x_{1}, x_{2}, x_{3}\right)=\alpha \phi\left(x_{2}, x_{3}\right) \\
& u_{2}\left(x_{1}, x_{2}, x_{3}\right)=-\alpha x_{1} x_{3} \\
& u_{3}\left(x_{1}, x_{2}, x_{3}\right)=\alpha x_{1} x_{2}
\end{aligned}
$$

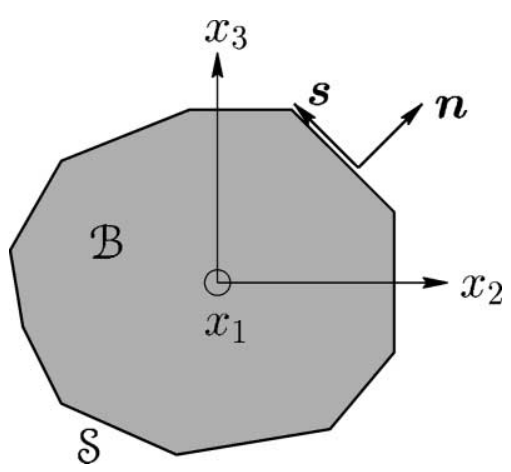

Fig. 1. Cross-section of bar considered in the augmented continuum theory of torsion. 
where $\phi$ is the warping function. The only non-vanishing strain components derived from these displacements are

$$
\epsilon_{12}\left(x_{2}, x_{3}\right)=\frac{\alpha}{2}\left(\frac{\partial \phi}{\partial x_{2}}-x_{3}\right), \quad \epsilon_{13}\left(x_{2}, x_{3}\right)=\frac{\alpha}{2}\left(\frac{\partial \phi}{\partial x_{3}}+x_{2}\right)
$$

The bulk material is assumed to be a linear elastic solid and thus the bulk stresses are related to the strain via

$$
\sigma_{12}=2 G \epsilon_{12}, \quad \sigma_{13}=2 G \epsilon_{13}
$$

where $G$ is an appropriate shear modulus (for example, if the ' 1 ' direction corresponds to the $\langle 100\rangle$ direction in an FCC crystal, $G=C_{44}$ ). The only non-trivial equilibrium equation in the bulk is

$$
\frac{\partial \sigma_{12}}{\partial x_{2}}+\frac{\partial \sigma_{13}}{\partial x_{3}}=0
$$

Eq. (15) is identically satisfied on the introduction of a stress function $\psi$ (after Prandtl) such that

$$
\sigma_{12}=G \alpha \frac{\partial \psi}{\partial x_{3}}, \quad \sigma_{13}=-G \alpha \frac{\partial \psi}{\partial x_{2}}
$$

On substituting the expressions for the stresses (16) in (14) and using (13) it is found that the stress function satisfies

$$
\frac{\partial^{2} \psi}{\partial x_{2}^{2}}+\frac{\partial^{2} \psi}{\partial x_{3}^{2}}=-2
$$

in the bulk.

The surface is parametrised by the coordinate $x_{1}$ and distance $s$ measured along the tangent vector $s$. The pertinent component of surface stress is $\tau_{1 s}$ where $s$ stands for the $s$ direction in Fig. 1 and satisfies the surface equilibrium equation (5)

$$
\frac{\partial \tau_{1 s}}{\partial s}+f_{1}=0
$$

The second surface equilibrium condition (6) is identically satisfied since $\kappa_{\alpha \beta}=0$. The "surface body force" $f_{1}$ is given as

$$
f_{1}=-t_{1}=-\left(\sigma_{12} n_{2}+\sigma_{13} n_{3}\right)=-G \alpha\left(\frac{\partial \psi}{\partial x_{3}} n_{2}-\frac{\partial \psi}{\partial x_{2}} n_{3}\right)=-G \alpha\left(\frac{\partial \psi}{\partial x_{3}} s_{3}+\frac{\partial \psi}{\partial x_{2}} s_{2}\right)=-G \alpha \frac{\partial \psi}{\partial s}
$$

The surface stress $\tau_{1 s}$ is related to the surface strain as

$$
\tau_{1 s}=2 S \epsilon_{1 s}
$$

where $S$ is an appropriate surface shear modulus ( $\tau_{1 s}^{0}$ can be taken to be zero with out loss of generality), i.e.,

$$
\tau_{1 s}=2 S \epsilon_{1 s}=2 S\left(-\epsilon_{12} n_{3}+\epsilon_{13} n_{2}\right)=2 S\left(-\frac{\sigma_{12}}{2 G} n_{3}+\frac{\sigma_{13}}{2 G} n_{2}\right)=-S \alpha\left(\frac{\partial \psi}{\partial x_{2}} n_{2}+\frac{\partial \psi}{\partial x_{3}} n_{3}\right)=-S \alpha \frac{\partial \psi}{\partial n}
$$

Substituting (19) and (21) in (18) the boundary condition on $\mathscr{S}$ for $\psi$ is obtained as

$$
\frac{\partial}{\partial s}\left(\psi+\frac{S}{G} \frac{\partial \psi}{\partial n}\right)=0, \quad \Rightarrow \quad \psi+\frac{S}{G} \frac{\partial \psi}{\partial n}=C
$$


where $C$ is a constant along the boundary. It is assumed that $S$ does not depend on $s$, i.e., all the bounding planes are assumed to be crystallographically equivalent.

The torsional rigidity of the bar can be computed as follows. The torque $T$ has contributions from the bulk $\mathscr{B}$ and the surface $\mathscr{S}$ and is given as

$$
\begin{aligned}
T & =\int_{\mathscr{B}}\left(x_{2} \sigma_{13}-x_{3} \sigma_{12}\right) \mathrm{d} x_{2} \mathrm{~d} x_{3}+\int_{\mathscr{S}} \tau_{1 s}\left(x_{2} n_{2}+x_{3} n_{3}\right) \mathrm{d} s \\
& =2 G \alpha \int_{\mathscr{B}} \psi \mathrm{d} x_{2} \mathrm{~d} x_{3}-G \alpha \int_{\mathscr{S}}\left(\psi+\frac{S}{G} \frac{\partial \psi}{\partial n}\right) \mathrm{d} s
\end{aligned}
$$

Evidently, the constant $C$ can be chosen to be zero. The torsional rigidity $D$ can be obtained by solving the mixed boundary value problem

$$
\begin{aligned}
& \frac{\partial^{2} \psi}{\partial x_{2}^{2}}+\frac{\partial^{2} \psi}{\partial x_{3}^{2}}=-2 \quad \text { in } \mathscr{B} \\
& \psi+\frac{S}{G} \frac{\partial \psi}{\partial n}=0 \quad \text { on } \mathscr{S}
\end{aligned}
$$

and substituting for $\psi$ in the expression

$$
D=2 G \int_{\mathscr{B}} \psi \mathrm{d} x_{2} \mathrm{~d} x_{3}
$$

Clearly, the theory reduces to the standard theory of torsion when $S$ is set to zero. It is also clear that the key parameter that determines the atomistic surface effects that affect the torsional rigidity is the material length-scale $h_{0}$ defined by the ratio of the surface shear modulus $S$ and the bulk shear modulus $G$, i.e., by

$$
h_{0}=\frac{S}{G}
$$

In Section 2.3, a perturbative solution to the boundary value problem (24) will be developed and a general formula for the size-dependent torsional rigidity will be obtained.

\subsection{Perturbative solution}

The general perturbative solution will be developed in a non-dimensional form. To this end, the geometry of the cross-section of the bar is assumed to be characterised by a length scale $a$ (for example, if the cross-section is a square, $a$ can be chosen as one half of the side of the square). The following nondimensional quantities are introduced

$$
\Psi=\frac{\psi}{a^{2}}, \quad \xi=\frac{x_{2}}{a}, \quad \eta=\frac{x_{3}}{a}
$$

In terms of these non-dimensional quantities, the boundary value problem (24) can be recast as

$$
\begin{aligned}
& \nabla^{2} \Psi=-2 \text { in } \mathscr{B} \\
& \Psi+\beta \frac{\partial \Psi}{\partial n}=0 \text { on } \mathscr{S}
\end{aligned}
$$

where $\nabla^{2}=\partial^{2} / \partial \xi^{2}+\partial^{2} / \partial \eta^{2}$ and the rigidity is

$$
\frac{D}{G a^{4}}=2 \int_{\mathscr{B}} \Psi \mathrm{d} \xi \mathrm{d} \eta
$$


with

$$
\beta=\frac{h_{0}}{a},
$$

the non-dimensional parameter that governs the extent of surface effects. When $\beta=0$, the boundary value problem is solved by the non-dimensional stress function $\Psi_{0}$ and the torsional rigidity is

$$
\frac{D_{\mathrm{c}}}{G a^{4}}=2 \int_{\mathscr{B}} \Psi_{0} \mathrm{~d} \xi \mathrm{d} \eta
$$

where the subscript ' $\mathrm{c}$ ' is used to denote that standard continuum value of the torsional rigidity.

When $\beta \neq 0$, the non-dimensional stress function can be expanded in a perturbative series in $\beta$ as

$$
\Psi=\Psi_{0}+\beta \Psi_{1}+\beta^{2} \Psi_{2}+\cdots
$$

Since $\Psi$ solves the boundary value problem (28), it follows that

$$
\beta \nabla^{2} \Psi_{1}+\beta^{2} \nabla^{2} \Psi_{2}+\cdots=0
$$

in $\mathscr{B}$ and

$$
\beta\left(\Psi_{1}+\frac{\partial \Psi_{0}}{\partial n}\right)+\beta^{2}\left(\Psi_{2}+\frac{\partial \Psi_{1}}{\partial n}\right)+\cdots=0
$$

on $\mathscr{S}$. Since the perturbative expansion (32) is valid for all values of $\beta$, (33) and (34) imply that

$$
\begin{array}{cc}
\nabla^{2} \Psi_{1}=0 & \text { on } \mathscr{B} \text { with } \Psi_{1}=-\frac{\partial \Psi_{0}}{\partial n} \text { on } \mathscr{S} \\
\nabla^{2} \Psi_{2}=0 & \text { on } \mathscr{B} \text { with } \Psi_{2}=-\frac{\partial \Psi_{1}}{\partial n} \text { on } \mathscr{S} \\
\vdots & \\
\nabla^{2} \Psi_{k}=0 & \text { on } \mathscr{B} \text { with } \Psi_{k}=-\frac{\partial \Psi_{k-1}}{\partial n} \text { on } \mathscr{S}
\end{array}
$$

which is a sequence of Dirichlet boundary value problems. Thus, the solution of the boundary value problem (28) can be obtained to any desired accuracy in the parameter $\beta$, and the torsional rigidity obtained as

$$
\frac{D}{G a^{4}}=A_{0}+A_{1} \beta+A_{2} \beta^{2}+\cdots
$$

where

$$
A_{k}=2 \int_{\mathscr{B}} \Psi_{k} \mathrm{~d} \xi \mathrm{d} \eta
$$

are constants that depend only on the shape of the cross-section. Since $D_{\mathrm{c}} / G a^{4}=A_{0}$, a general expression for the size dependence of the torsional rigidity can be derived as

$$
\frac{D-D_{\mathrm{c}}}{D_{\mathrm{c}}}=\frac{A_{1}}{A_{0}} \beta+\frac{A_{2}}{A_{0}} \beta^{2}+\cdots
$$

For cross-sections of a given shape, the constants $A_{k}$ can be calculated once and for all and the formula (40) along with (26) and (30) can be used to predict the size dependence of the torsional rigidity. Atomistic inputs are required only in providing values for $S$ and $G$. It will be seen in the following sections that the 
value of $\beta$ in real systems is less that 0.5 and the most important contribution is from the first term on the right hand side of (40), i.e.,

$$
\frac{D-D_{\mathrm{c}}}{D_{\mathrm{c}}} \approx \frac{A_{1}}{A_{0}} \beta=\frac{A_{1}}{A_{0}} \frac{h_{0}}{a}
$$

\section{Atomistic simulations}

Atomistic simulations are carried out to validate the theory developed in the previous sections. The bars selected for study have a square cross-section (Fig. 2) made of selected FCC metals (Al, Ag, Cu, Ni) such that the ' 1 '-direction corresponds to the [ 1000$]$ crystallographic direction and ' 2 ' and ' 3 ' directions correspond to [ $\left[\begin{array}{lll}0 & 1 & 0\end{array}\right]$ and $\left[\begin{array}{lll}0 & 0 & 1\end{array}\right]$ crystallographic directions respectively, i.e., the bounding free surfaces are planes of the $\{100\}$ family.

Two sets of simulations are carried out. In the first set, the constitutive constants $G=C_{44}$ (bulk shear modulus) and $S$ the surface elastic constant for surface shear of the $\left\{\begin{array}{ll}10 & 0\end{array}\right\}$ surface are evaluated atomistically; these are the parameters required as input to the theory developed. In the second set of simulations the torsional rigidity is directly calculated using atomistic simulation of nanoscale torsion. These results are then compared with the theoretical results of torsional rigidity in Section 4.

The atomistic model used in the present study is the embedded atom method (EAM) developed by Daw and Baskes (1984). The elements $\mathrm{Ag}, \mathrm{Cu}$, and $\mathrm{Ni}$ are modeled using the EAM potentials of Oh and Johnson (1988) and Al is modeled with potentials developed by Ercolessi and Adams (1994).

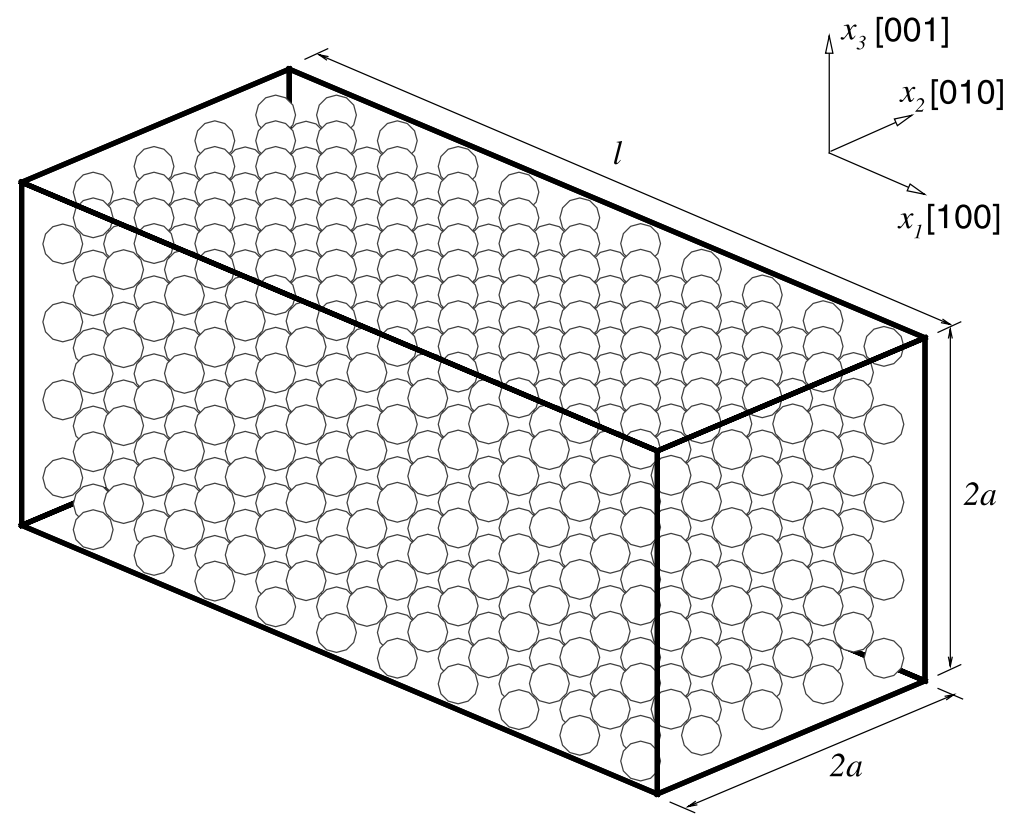

Fig. 2. Atomistic system used for the simulation of torsional response. 


\subsection{Determination of surface elastic constants}

The surface elastic constants are determined as follows. A block of atoms are stacked in an FCC crystal lattice such that the coordinate planes are of the $\left\{\begin{array}{lll}0 & 0 & 0\end{array}\right.$ family. Periodic boundary conditions are imposed in the ' 1 ' and ' 2 ' directions alone simulating bounding free surfaces of $\left\{\begin{array}{lll}1 & 0\end{array}\right\}$ family in the ' 3 ' direction. The positions of the atoms are changed to correspond to a deformation gradient tensor $\boldsymbol{F}=\boldsymbol{I}+\epsilon \boldsymbol{e}_{1} \otimes \boldsymbol{e}_{2}$ that produces a simple shear by amount $\epsilon$ ( $\boldsymbol{I}$ is the identity tensor, and $\boldsymbol{e}_{\mathrm{i}}$ are the orthonormal basis vectors). The potential energy of this atomistic system is minimised and the minimised total energy is calculated. To compute the surface energy, the elastic energy stored in the bulk is subtracted from the calculated total energy. On performing this simulation for various values of $\epsilon$, the surface energy $\gamma$ is obtained as a function of $\epsilon$, and the surface elastic constant is calculated by numerical differentiation of this function. It is noted that to compute the bulk elastic constant $G\left(=C_{44}\right)$ a similar procedure can be adopted with periodicity imposed in the ' 3 ' direction as well. The results of these simulations are shown in Table 1.

\subsection{Atomistic simulations of torsional response}

Atomistic simulations used to calculate the torsional rigidities are performed as follows. The simulation box $\left(0 \leqslant x_{1} \leqslant \ell,\left|x_{2}\right| \leqslant a,\left|x_{3}\right| \leqslant a\right)$ consists of a collection of atoms as shown in Fig. 2 with periodic boundary conditions imposed in the ' 1 ' direction with a periodic length of $\ell$. The atoms are then displaced according to the rotation tensor

$$
\boldsymbol{R}\left(x_{1}\right)=\boldsymbol{I}+\alpha x_{1}\left(\boldsymbol{e}_{3} \otimes \boldsymbol{e}_{2}-\boldsymbol{e}_{2} \otimes \boldsymbol{e}_{3}\right)
$$

where $\alpha$ is the twist per unit length ( $\alpha$ is chosen such that $\alpha \ell \ll 1$, so that the infinitesimal rotation tensor is sufficiently accurate). With this initial condition, the atomistic potential energy of the system is minimised. The energy and force computation during the minimisation process is carried forth as follows. To compute the energy of an atom (say $i$ ) near $x_{1}=\ell$ the position vector of its periodic neighbour (say $j$ ), found near $x_{1}=0$ in the box, is required. The position vector of the periodic image of atom $j$ is obtained by applying the rotation tensor $\boldsymbol{R}(\ell)$ to the vector $\boldsymbol{x}(j)+\ell \boldsymbol{e}_{1}$ where $\boldsymbol{x}(j)$ is the position vector of atom $j$ in the simulation box. Similarly the periodic neighbour $j$ of an atom $i$ near $x_{1}=0$ can be obtained by applying the inverse of the rotation tensor $\boldsymbol{R}(\ell)$. This procedure ensures that the periodicity in the '1' direction is maintained, while keeping the bar in a twisted position with twist per unit length $\alpha$. The size $\ell$ of the box was chosen to be greater than twice the cutoff radius of the potential.

Using the procedure above, simulations are carried out for various values of $\alpha$ and the minimised atomistic total energy $E$ in the simulation box is obtained as a function of $\alpha$. The torsional rigidity is calculated using the formula

$$
D=\frac{1}{\ell} \frac{\partial^{2} E}{\partial \alpha^{2}}
$$

Table 1

Properties of materials calculated using EAM potentials

\begin{tabular}{lllll}
\hline Material & $a_{0}(\AA)$ & $C_{44}\left(\mathrm{eV} / \AA^{3}\right)$ & $S\left(\mathrm{eV} / \AA^{2}\right)$ & $h_{0}(\AA)$ \\
\hline $\mathrm{Al}$ & 4.032 & 0.229 & 0.481 & 2.099 \\
$\mathrm{Ag}$ & 4.090 & 0.292 & 0.248 & 0.849 \\
$\mathrm{Cu}$ & 3.615 & 0.474 & 0.429 & 0.906 \\
$\mathrm{Ni}$ & 3.520 & 0.808 & 0.763 & 0.945 \\
\hline
\end{tabular}

$a_{0}$, the lattice parameter, $C_{44}$, the bulk shear modulus, $S$, the surface shear modulus and $h_{0}=S / C_{44}$. 
The correctness of this procedure is ascertained by choosing various values of $\ell$ for the periodic distance and computing $D$ using the above procedure. It is found that $D$ is insensitive to the choice of $\ell$.

\section{Results and discussion}

\subsection{Theoretical results for square bars}

\subsubsection{Exact solution}

The exact solution of the boundary value problem (24) for the case of a square bar is obtained in this section. The square section is assumed to be of side $2 a$ and the solution for the boundary value problem is obtained in the non-dimensional form (28) where the square occupies the region $|\xi| \leqslant 1,|\eta| \leqslant 1$. A straightforward analysis gives that

$$
\Psi(\xi, \eta)=8 \sum_{n=1}^{\infty} \frac{\sin k_{n}}{k_{n}^{2}\left(2 k_{n}+\sin 2 k_{n}\right)} \cos k_{n} \xi\left(1-\frac{\cosh k_{n} \eta}{\cosh k_{n}+\beta k_{n} \sinh k_{n}}\right)
$$

where $k_{n}$ is the $n$th root of the equation

$$
\cos k-\beta k \sin k=0
$$

The non-dimensional theoretical torsional rigidity (29) is obtained as

$$
\frac{D}{G a^{4}}=64 \sum_{n=1}^{\infty} \frac{\sin ^{2} k_{n}}{k_{n}^{3}\left(2 k_{n}+\sin 2 k_{n}\right)}\left(1-\frac{\sinh k_{n}}{k_{n}\left(\cosh k_{n}+\beta k_{n} \sinh k_{n}\right)}\right)
$$

For the purpose of comparison with atomistic simulations, the non-dimensional warping function $\Phi=\phi / a^{2}$ is also derived:

$$
\Phi(\xi, \eta)=\xi \eta-8 \sum_{n=1}^{\infty} \frac{\sin k_{n}}{k_{n}^{2}\left(2 k_{n}+\sin 2 k_{n}\right)\left(\cosh k_{n}+\beta k_{n} \sinh k_{n}\right)} \sin k_{n} \xi \sinh k_{n} \eta
$$

\subsubsection{Perturbative solution}

The function $\Psi_{0}$ is obtained by setting $\beta=0$ in the exact solution (44). The function $\Psi_{1}$ is obtained by solving the Dirichlet problem (35) as

$$
\Psi_{1}(\xi, \eta)=4 \sum_{n=0}^{n=\infty} \frac{\sin q_{n}}{q_{n}^{2}} \frac{\tanh q_{n}}{\cosh q_{n}}\left(\cos q_{n} \xi \cosh q_{n} \eta+\cosh q_{n} \xi \cos q_{n} \eta\right)
$$

where $q_{n}=(2 n-1) \pi / 2$. The constant $A_{1}$ can be calculated as

$$
A_{1}=64 \sum_{n=1}^{\infty} \frac{\sin ^{2} q_{n} \tanh ^{2} q_{n}}{q_{n}^{4}}
$$

The numerical values of $A_{0}$ (which is calculated from (46) with $\beta=0$ ) and $A_{1}$ are

$$
A_{0}=2.2492, \quad A_{1}=8.9969
$$

Thus,

$$
D \approx A_{0}+A_{1} \beta
$$




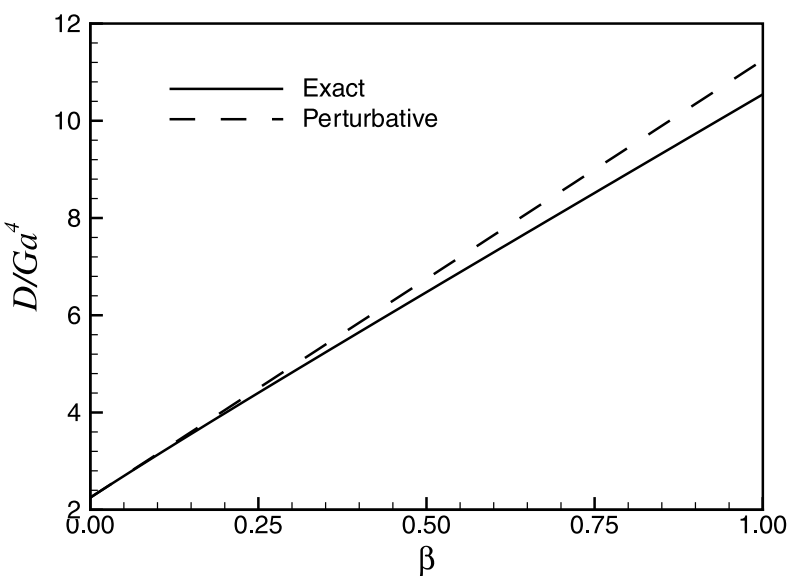

Fig. 3. Comparison of the exact (46) and perturbative (51) results for the torsional rigidity.

and

$$
\frac{D-D_{\mathrm{c}}}{D_{\mathrm{c}}} \approx \frac{A_{1}}{A_{0}} \beta=4 \beta=4 \frac{h_{0}}{a}
$$

A comparison between the exact result (46) and the perturbative result (51) is shown in Fig. 3. It is evident that the perturbative result is a very good approximation of the exact result; even when $\beta=1$ (which is much larger than that which would appear in systems considered here), the error is only $6.5 \%$. Thus the perturbative result in the form of (52) is used in comparisons with atomistic results.

\subsection{Comparison of atomistic and theoretical results}

Fig. 4 shows a comparison between the non-dimensional torsional rigidity computed atomistically and that predicted by the augmented continuum theory (52). Several points may be noted:

1. It is clear that the atomistically calculated torsional rigidity differs significantly from the predictions of standard continuum theory. In fact, for an Al bar of width $5 \mathrm{~nm}$ the torsional rigidity is about $50 \%$ larger.

2. The non-dimensional difference in torsional rigidity computed atomistically scales very closely as $(1 / a)$ in all the metals.

3. The atomistically computed values are accurately predicted by the theoretical values for $\mathrm{Al}$ and $\mathrm{Ni}$ (within $10 \%$ ), while the agreement is fair for $\mathrm{Ag}$ and $\mathrm{Cu}$ (within $30 \%$ ).

4. In all cases, the atomistically computed values are larger than the theoretical values, although to varying degrees in different metals.

It may be argued that the reason for the atomistic values being greater than the theoretical values is the neglect of corner effects in the theoretical analysis. A simple dimensional analysis indicates that corner effects must scale as $1 / a^{2}$; but the atomistic results scale very closely as $1 / a$. Thus it is clear that corners play a secondary role in the systems considered here. A more plausible reason for the difference in the theoretical values and simulation results is the assumption that the surface energy $\gamma$ depends only on surface strain. In reality the surface energy can also depend on the surface curvature strain $b_{\alpha \beta}$, i.e., the difference between the 

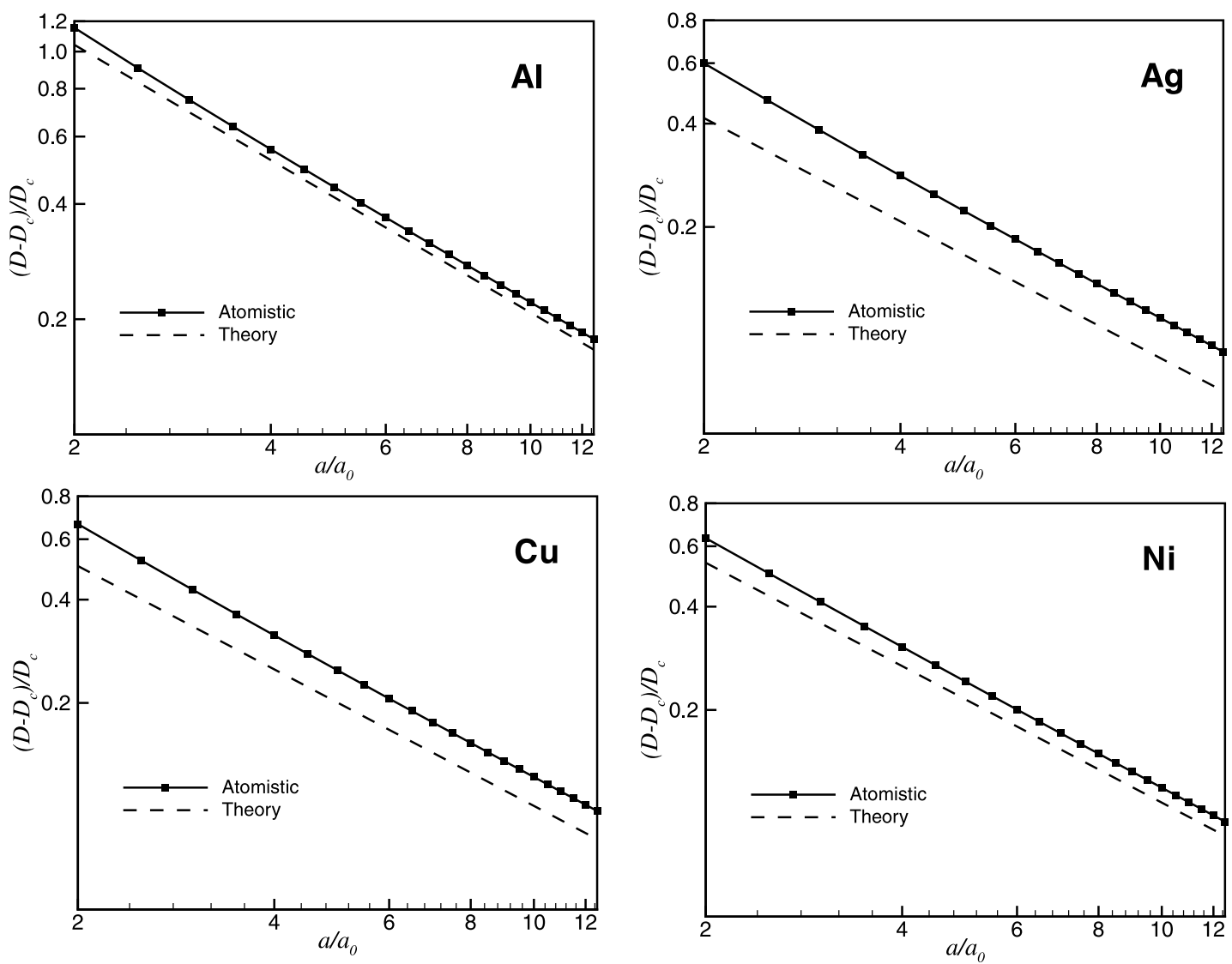

Fig. 4. Comparison of the results of atomistic simulations with theoretical results. $a_{0}$ is the lattice parameter.

deformed curvature and the original curvature of the surface. Thus the surface energy must be a function of both the surface strain tensor and the surface curvature strain tensor; mathematically, $\gamma=\gamma\left(\epsilon_{\alpha \beta}, b_{\alpha \beta}\right)$. This idea can be expressed more physically; in the present model, the surface is treated as a membrane, while a more physically realistic model will be that of a shell with bending stiffness. Additional evidence in support of this argument is that in the case of bending of plates treated by Miller and Shenoy (2000) the augmented continuum theory differs by the order of $30 \%$ with the atomistic simulations. The plates treated did not have corners and this effect can only be attributed to the neglect of the bending energy of the surfaces. Clearly this problem requires a more elaborate theoretical framework and will be taken up for study in future.

The atomistic simulations of torsion not only allow for the computation of the torsional rigidity but also provide data for the warping of atomic planes. From the positions of atoms at the configuration of minimum energy, the values of non-dimensional warping $\Phi$ are calculated. The atomistically computed values of the non-dimensional warping must be independent of the value of $\alpha$ (twist per unit length) according to the theory. This is indeed found in the simulations, and provides a further check for the theory. The atomistically simulated warping displacements are then compared with the theoretically predicted values (47). The result of such an exercise is plotted in Fig. 5. It is evident that the predicted warping is in excellent agreement with the atomistic result. 


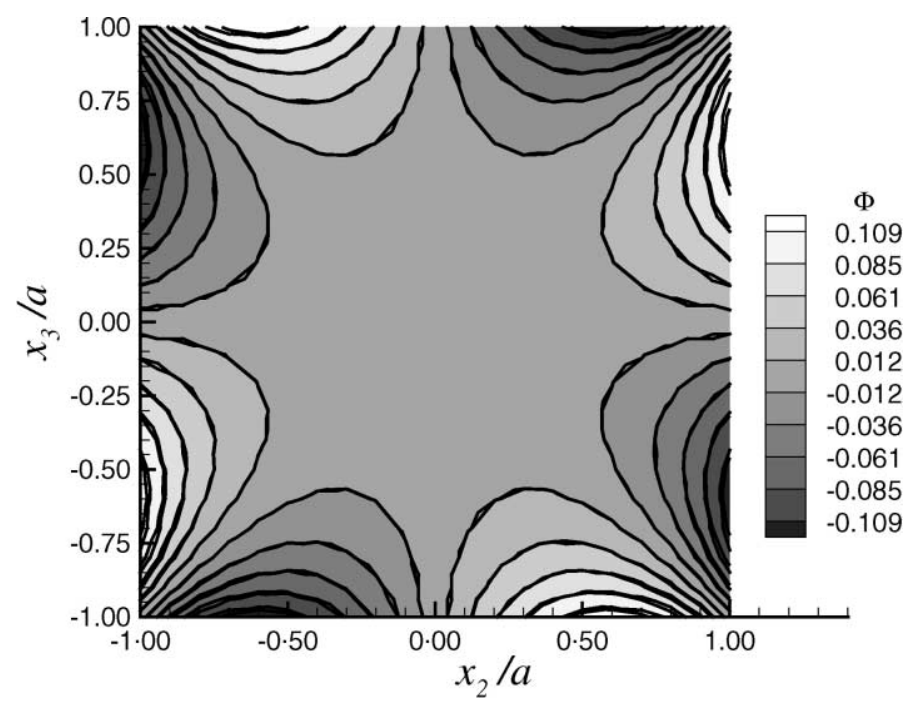

Fig. 5. Comparison of atomistically simulated and theoretical warping function $\Phi$. The solid lines are the contours of the atomistic result while the dashed lines correspond to the theoretical calculation (47). The atomistic result is for $\mathrm{Al}$ with $a / a_{0}=5$ and the theoretical result corresponds to $\beta=0.1$.

\section{Conclusions}

A general framework for the prediction of rigidities of nanoscale structural elements has been developed and applied to the case of the nanoscale bars in torsion. The key premise of this theory is that the heterogeneities present in such systems can be modeled as surface effects in an augmented continuum theory. A development of this idea reveals that the material length scale that governs the size dependence of the rigidity is the ratio of the surface elastic constant to the bulk elastic constant. The augmented theory of torsion developed here is compared with direct atomistic calculations of bars of various metals and is found to be satisfactory. The author is not aware of any work that reports experimentally measured torsional rigidities of nanosized bars - indeed, atomistic simulations are used as numerical experiments. Given the advances in nanotechnology, such experiments are expected to be performed in the near future.

The use of this theory is envisaged as follows. The bulk elastic constants and the surface elastic constants (for various surfaces) of materials of interest can be calculated and tabulated. The expressions for the constants that appear in the perturbative expansion in size dependence of the torsional rigidity can be worked out for a host of cross-sectional shapes once and for all. A collection of such information will be useful for the designers of nanomechanical systems in that the need for direct atomistic simulations of nanoscale structures is obviated. The work of Miller and Shenoy (2000) along with the present work provide a complete framework for the prediction of rigidities of nanoscale structural elements in extension, flexure and torsion.

Several points for future work are noted. The atomistic model used in this study, EAM, is known to be inaccurate with applied to interfacial properties. This, of course, does not invalidate the present work since the parameters used in the theory and the simulation results are obtained from the same EAM model. To obtain accurate values of surface elastic constants more sophisticated atomistic models such as density functional theory may be applied. Another important point to be noted is that thermal effects are not accounted in the calculation of the surface elastic constants. Also, the model developed here treats the surface as a membrane while a more realistic model will have to additionally account for the bending stiffness of the surface. Results of investigations along the afore mentioned directions will be reported in future publications. 


\section{Acknowledgements}

The author wishes to thank Ron Miller and Rob Phillips for useful discussions. Partial support for this work provided by CDAC, Poona, India, is acknowledged.

\section{References}

Cammarata, R.C., 1994. Surface and interface stress effects in thin films. Progress in Surface Science 46 (1), 1-38.

Dai, H., Hafner, J.H., Rinzler, A.G., Colbert, D.T., Smalley, R.E., 1996. Nanotubes as nanoprobes in scanning probe microscopy. Nature 384, 147-150.

Daw, M.S., Baskes, M.I., 1984. Embedded-atom method: derivation and application to impurities, surfaces, and other defects in metals. Physical Review B 29, 6443-6453.

Ercolessi, F., Adams, J.B., 1994. Interatomic potentials from first-principles calculations-the force-matching method. Europhysics Letters 26, 583.

Gao, R., Wang, Z.L., Bai, Z., de Heer, W.A., Dai, L., Gao, M., 2000. Nanomechanics of individual carbon nanotubes from pyrolitically grown arrays. Physical Review Letters 85, 622-625.

Garg, A., Sinnott, S.B., 1998. Effect of chemical functionalization on the mechanical properties of carbon nanotubes. Chemical Physics Letters 295, 273-278.

Garg, A., Han, J., Sinnott, S.B., 1998. Interactions of carbon-nanotube proximal probe tips with diamond and graphene. Physical Review Letters 81 (11), 2260-2263.

Gurtin, M.E., Murdoch, A.I., 1975. A continuum theory of elastic material surfaces. Archive for Rational Mechanics and Analysis 57 (4), 291-323.

Kuzumake, T., Miyazawa, K., Ichinose, H., Ito, K., 1998. Processing of carbon nanotube reinforced aluminum composite. Journal of Materials Research 13 (9), 2445-2446.

Miller, R.E., Shenoy, V.B., 2000. Size-dependent elastic properties of nanosized structural elements. Nanotechnology 11, $139-147$.

Oh, D.J., Johnson, R.A., 1988. Simple embedded atom method model for fcc and hep metals. Journal of Materials Research 3, 471-478.

Poncharal, P., Wang, Z., Ugarte, D., de Heer, W.A., 1999. Electrostatic deflections and electromechanical resonances of carbon nanotubes. Science 283, 1513-1516.

Rice, J.R., Chuang, T.-J., 1981. Energy variations in diffusive cavity growth. Journal of the American Ceramic Society 64 (1), 46-53.

Robertson, D.H., Brenner, D.W., Mintmire, J., 1992. Energetics of nanoscale graphitic tubules. Physical Review B 45 (21), $12592-$ 12595 .

Roukes, M.L., 2000. Nanoelectromechanical Systems. Available from <http://arXiv.org/abs/cond-mat/0008187>.

Sheehan, P.E., Lieber, C.M., 1996. Nanotribology and nanofabrication of $\mathrm{MoO}_{3}$ structures by atomic force microscopy. Science 272, $1158-1161$.

Sokolnikoff, I.S., 1956. Mathematical Theory of Elasticity. McGraw-Hill, New York.

Terrones, M., Grobert, N., Hsu, W., Zhu, Y., Hu, W., Terrones, H., Hare, J., Kroto, H., Walton, D., 1999. Advances in the creation of filled nanotubes and novel nanowires. Materials Research Society Bulletin 24 (8), 43-49.

Wong, E., Sheehan, P.E., Lieber, C.M., 1997. Nanobeam mechanics: elasticity, strength, and toughness of nanorods and nanotubes. Science 277, 1971-1975.

Yakobson, B.I., Smalley, R., 1997. Fullerene nanotubes: $C_{1,000,000}$ and beyond. American Scientist 85 (4), 324-337.

Yannouleas, C., Landman, U., Herlert, A., Schweikhard, L., 2001. Trianionic gold clusters. The European Physical Journal D 16, 81-86. 